\title{
Determination of Effective Elastic Properties of Microcracked Rocks Based on Asymptotic Approximation
}

\author{
J.D. Zhao Centre for Geotechnical and Materials Modelling, The University of Newcastle, Australia \\ S.W. Sloan Centre for Geotechnical and Materials Modelling, The University of Newcastle, Australia \\ J.P. Carter Centre for Geotechnical and Materials Modelling, The University of Newcastle, Australia
}

\begin{abstract}
In this paper, we present a study on the effective elastic properties of finely fractured rock based on the concept of energy equivalence. The effective elastic properties a rock body weakened by many penny-shaped microcracks, such as the apparent Young's modulus, shear modulus and Poisson's ratio, are particularly important in many applications. To determine these macroscopic parameters, we first adopt the dilute solution approach outlined in Kachanov (1992), where a crack compliance tensor is defined and used extensively to express the energy perturbation caused by the presence of the cracks. In parallel, a selfconsistent method is employed where an asymptotic form of the Eshelby's tensor (Eshelby, 1957) is developed to treat a penny-shaped microcrack as an extreme case of a spheroid-shaped inhomogeneity. The asymptotic approximation permits the local Eshelby tensor as well as its global expression to be derived analytically, and to be further used to construct an equivalent eigenstrain problem based on energy equivalence. The effective values for the parameters of interest can then be evaluated. The formulation and results obtained through the asymptotic self-consistent approach are compared to those predicted using a non-interacting scheme.
\end{abstract}

\section{Introduction}

One of the classic problems in solid mechanics, geophysics and material science is the determination of the effective elastic properties of cracked solids. It is particularly important for constitutive modelling of brittle microcracked materials such as rocks containing large quantities of microcracks. The elastic properties of cracked rocks depend on a number of factors: the mineral properties and their distribution, the porosity type, magnitude and distribution, and the state of saturation of the rock. There are two major theoretical approaches in the literature addressing the problem of effective elastic moduli of cracked rocks. The first approach includes the effective medium theories that assume separate pores and cracks that may or may not be inter-connected within the rock; the second is the poroelastic theory which assumes that significant portions of the pores and cracks are inter-connected. The poroelastic theories were pioneered by the constitutive equations developed by Biot (1941), which are essentially phenomenological in nature and thus do not require characterisation of the matrix and pore space geometry. In contrast, the effective medium theories generally require parameters characterising the pore shape and distribution and can be traced back as early as the classical bounds provided by Voigt (1928) and Reuss (1929) and Hashin and Shtrikman (1961, 1962). These pioneering works were motivated by microcracking in either metals or in rocks and the effective constants were derived for the isotropic case of randomly oriented cracks of circular/penny shapes in the non-interaction approximation (Bristow, 1960; Walsh 1965a; 1965b). A major flaw of these early noninteraction studies is that, when the crack densities are so high that interactions among cracks become significant, the non-interaction predictions will inevitably lose their accuracy. To account for crack interactions, numerous improved approximate schemes have been proposed. See Kachanov (2007) for a recent review. Amongst them are the well-known self-consistent scheme (Budiansky and O'Connell, 1976), the differential method (Vavakin and Salganik, 1975; Norris, 1985; Hashin, 1988; Zimmerman, 1991), the Mori-Tanaka method (Mori and Tanaka, 1973; Benveniste, 1987), and the non-interacting method (Kachanov, 1992).

A common feature in most of these latter schemes is that they consider the interactions by placing noninteracting cracks into an "effective environment". Many early studies focus on a general solid with a matrix 
containing inclusions, from which the problem of effective elastic moduli of cracked solids can be regarded as a limiting case. In this limit, it is assumed the inclusions shrink to surfaces and meanwhile their elastic moduli tend to zero. As remarked in a review by Kachanov (1992), however, there are some difficulties pertaining to this approach, such as the dependence of the transition order of the limit, the definition of the crack density parameter which can not take into account the crack shape, and problems associated with some existing approaches in achieving the degeneration. Predictions by these various schemes also diverge significantly as crack density increases. The self-consistent scheme gives a strong softening effect of the interaction, while the differential scheme gives rise to a substantially milder softening effect. The MoriTanaka scheme for materials with interacting inclusions predicts no interaction effect at all when applied to cracked solids. As far as cracks are concerned, the seminal Hashin and Shtrikman (1963) bounds fail to provide any guidance. The Hashin and Shtrikman (1963) bound on the effective modulus $\kappa_{*}$ of a threedimensional (3D) two-phase composite is:

$$
c_{1} \kappa_{1}+c_{2} \kappa_{2}-\frac{c_{1} c_{2}\left(\kappa_{1}-\kappa_{2}\right)^{2}}{c_{1} \kappa_{2}+c_{2} \kappa_{1}+4 \mu_{2} / 3} \leq \kappa_{*} \leq c_{1} \kappa_{1}+c_{2} \kappa_{2}-\frac{c_{1} c_{2}\left(\kappa_{1}-\kappa_{2}\right)^{2}}{c_{1} \kappa_{2}+c_{2} \kappa_{1}+4 \mu_{1} / 3}
$$

where $\kappa_{1}, \kappa_{2}, \mu_{1}$ and $\mu_{2}\left(\mu_{2} \leq \mu_{1}\right)$ are the phase bulk and shear moduli, and $c_{1}$ and $c_{2}$ are the phase volume fractions. Now consider the case of cracks where the quantities $\kappa_{2}, \mu_{2}$ and $c_{2}$ all tend to zero, so that the upper bound in the Hashin and Shtrikman (1963) expression degenerates into the following trivial one: $\kappa_{*} \leq \kappa_{1}$, with the lower bound being indeterminable directly from Equation (1) (although from physical consideration it should be zero). Comprehensive comparisons and comments of the various approaches when applied to cracked solids can be found in Kachanov (1992).

In view of the above, direct approaches have been suggested by Kachanov (1992) to solve the problem of effective moduli of cracked solids. In this paper we employ two different approaches: one is the noninteracting scheme outlined in Kachanov (1992) and based on a crack compliance tensor; and the other is a self-consistent approach based on an asymptotic approximation of the Eshelby's tensor. Predictions of the effective moduli obtained using the two methods are compared with each other as well as with an approximate analytical solution using a differential scheme.

\section{Non-interacting scheme based on crack compliance tensor}

\subsection{Crack density parameter}

Budiansky and O'Connell (1976) defined the two-dimensional (2D) scalar crack density $\beta$ for a microcracked solid as:

$$
\beta=N\left\langle l^{2}\right\rangle
$$

where $N$ is the number of microcracks per unit area in the plane of interest, $l$ is the half length of a $2 \mathrm{D}$ slitlike microcrack, and $\left\langle l^{2}\right\rangle$ stands for the average of $l^{2}$ overall microcracks. The rock matrix is assumed to be isotropic.

Kachanov $(1980 ; 1987)$ defined the following symmetric second-order crack density tensor for the general case of 3D solids with penny-shaped cracks:

$$
\boldsymbol{\alpha}=\frac{1}{V} \sum_{k} \gamma_{k} \mathbf{n}_{k} \mathbf{n}_{k}
$$

where $V$ is the volume of averaging, $\mathbf{n}_{k}$ is the unit normal to the $k$ th crack and $\gamma_{k}$ is a weighting factor characterising the contribution of the $k$ th crack to $\boldsymbol{\alpha}$ and depending on the physical problem of interest. In the case of the effective properties, $\gamma_{k}=r_{k}^{3}$, where $r_{k}$ is the radius of the $k$ th crack for the 3D solids containing the penny-shaped cracks. For a $2 \mathrm{D}$ solid with slit-like cracks, $\gamma_{k}=l_{k}^{2}$ where $l_{k}$ is the half-length 
of the $k$ th crack and $V$ now represents the area over which the averaging is conducted. Note that the trace of $\boldsymbol{\alpha}, \rho=t \boldsymbol{r} \boldsymbol{\alpha}$, coincides with the scalar crack density, as defined in Equation (2) for a general 2D case, and with the scalar crack density $\rho=N\left\langle l^{3}\right\rangle$ for the general case, which implies that $\boldsymbol{\alpha}$ represents a tensorial generalisation of $\rho$ accounting for the crack orientation statistics. The crack density plays a role roughly similar to the role of the volume fraction for two-phase composites.

\subsection{Crack compliance and the non-interacting scheme}

Kachanov $(1992 ; 1994)$ introduced a crack compliance tensor to characterise the crack opening displacement (COD) of a crack in a matrix under external force. A similar expression was first used by Hill (1963) for a more general case of arbitrary cavities. It is assumed that across the crack surfaces the displacements are discontinuous such that the strains are singular, which can be denoted by the following expression:

$$
\varepsilon_{i j}=M_{i j k l}^{0} \sigma_{k l}+\frac{1}{2} \sum_{k}\left(b_{i} n_{j}+b_{j} n_{i}\right)^{(k)} \delta\left(S^{(k)}\right)
$$

where the first term in the right-hand side, $M_{i j k l}^{0} \sigma_{k l}$, denotes the continuous part of the strain with $M_{i j k l}^{0}$ being the linear elastic compliance tensor of the matrix and $\sigma_{k l}$ the stresses acting on the k direction perpendicular to the 1 direction? $\delta(\cdot)$ is a delta function concentrated on the $k$ th crack's surfaces $s^{(k)}, \mathbf{b}=\mathbf{u}^{+}-\mathbf{u}^{-}$is the vector for the displacement jump across the crack surfaces (or COD) following Kachanov (1992), $\mathbf{n}$ is the unit normal to a crack. Both $\mathbf{n}$ and $\mathbf{b}$ are generally variable along cracks. Averaging the above expression over the volume of the material body $V$ and using the property of $\delta(\cdot)$ that the integral $\int_{V} f(x) \delta(s) d V$ can be reduced to the surface integral $\int_{S} f(x) d s$, we have:

$$
\begin{aligned}
\left\langle\varepsilon_{i j}\right\rangle & =M_{i j k l}^{0}\left\langle\sigma_{k l}\right\rangle+\frac{1}{2 V} \sum_{k} \int_{S^{(k)}}\left(b_{i} n_{j}+b_{j} n_{i}\right)^{(k)} d s \\
& =\left(M_{i j k l}^{0}+\Delta M_{i j k l}\right)\left\langle\sigma_{k l}\right\rangle=M_{i j k l}\left\langle\sigma_{k l}\right\rangle
\end{aligned}
$$

where $\Delta M_{i j k l}$ is the change in compliance due to cracks and $M_{i j k l}$ is the effective compliance.

It is assumed that the material body contains a statistically homogeneous field of cracks and is sufficiently large to be representative. In this case it can be reasonably hypothesised that the average stress is equal to the constant boundary traction stress: $\left\langle\sigma_{k l}\right\rangle=\sigma_{k l}^{0}=\sigma_{k l}^{\infty}$. Hereafter we omit the averaging signs for both stresses and strains. For flat cracks where the unit outer normal is constant for each crack, we have:

$$
\varepsilon_{i j}=M_{i j k l}^{0} \sigma_{k l}+\frac{1}{2 V} \sum_{k}\left(\left\langle b_{i}\right\rangle n_{j}+\left\langle b_{j}\right\rangle n_{i}\right)^{(k)} s^{(k)}
$$

It is thus observed that the contribution of a given crack to the overall strain is proportional to the product of the crack surface with COD. It was Hill (1963) who first proposed this type of expression for a more general case of arbitrary cavities. Hill (1963) expressed the cavity's contribution to the overall strain as an integral over the cavity surface:

$$
\Delta \varepsilon_{i j}=\frac{1}{2 V_{S}} \int_{S}\left(u_{i} n_{j}+u_{j} n_{i}\right) d s
$$


This reduces to the second term of Equation (6) when the cavities shrink to cracks. The elastic potential of a cracked solid can be obtained by:

$$
\begin{aligned}
P\left(\sigma_{i j}\right) & =\frac{1}{2} \sigma_{i j} M_{i j k l} \sigma_{k l}=\frac{1}{2} \sigma_{i j} M_{i j k l}^{0} \sigma_{k l}+\frac{1}{2 V} \sum_{k}\left(n_{i} \sigma_{i j}\left\langle b_{j}\right\rangle\right)^{(k)} s^{(k)} \\
& =P_{0}\left(\sigma_{i j}\right)+\Delta P
\end{aligned}
$$

where $P_{0}\left(\sigma_{i j}\right)$ is the potential of a matrix material without cracks, which has the following expression for an isotropic (no crack) material with Young's modulus $E_{0}$ and Poisson's ratio $v_{0}$ :

$$
P_{0}\left(\sigma_{i j}\right)=\frac{1+v_{0}}{2 E_{0}} \sigma_{i j} \sigma_{i j}-\frac{v_{0}}{2 E_{0}} \sigma_{k k}^{2}
$$

In a $2 \mathrm{D}$ case, $E_{0}$ is replaced with $E_{0}^{\prime}$ where $E_{0}^{\prime}=E_{0}$ for the plane stress case and $E_{0}^{\prime}=E_{0} /\left(1-v_{0}^{2}\right)$ for the plane strain case. $\Delta P$ in Equation (8) is the perturbation of the potential induced by the introduction of cracks into the material body, which is crucial in determining the effective moduli of the cracked rocks. To this end, Kachanov (1992) introduced a second rank tensor B to relate the uniform traction $\mathbf{t}$ applied at the crack surface and the resulting average crack opening displacement:

$$
\left\langle b_{j}\right\rangle=t_{i} B_{i j}
$$

which he called the crack compliance tensor. For an infinite material body, $\mathbf{B}$ depends on crack size, shape and the elastic properties of the matrix and, in the case of an anisotropic matrix, on the orientation of the crack with respect to the anisotropy axes of the matrix as well. In the case of a single crack in an infinite body with stress at infinity, Equation (10) can be rewritten as:

$$
\left\langle b_{j}\right\rangle=n_{l} \sigma_{i l} B_{i j}
$$

where $B_{i j}$ is a symmetric tensor. In a local coordinate frame $\left(n_{n}, n_{t}\right)$, where $n$ and $t$ are the in plane normal and tangential directions of the crack, the normal and shear components of COD produced by normal and shear tractions are denoted by $B_{n}$ and $B_{t}$, respectively. The off-diagonal components characterise coupling of the modes that is relevant for an anisotropic matrix and for non-circular cracks in an isotropic matrix.

For non-interacting cracks randomly distributed in an infinite isotropic solid, the compliance linearly depends upon the crack density parameter and the normal and shear modes are uncoupled. In the 2D case, B is proportional to a unit tensor:

$$
B_{i j}=\frac{\pi r}{E_{0}^{\prime}} \delta_{i j}
$$

where $r$ is the half-length of the crack. In this case, the compliances in the normal and shear directions are equal to each other. The perturbed potential $\Delta P$ in Equation (8) is now:

$$
\Delta P=\frac{\pi}{E_{0}^{\prime}} \sigma_{i j} \sigma_{j k} \alpha_{i k}
$$

where $\boldsymbol{\alpha}$ is defined in Equation (3). The elastic potential density $P$ is a function of $\sigma_{i j}$ and $\alpha_{i j}$ and $\Delta P$ is a simultaneous invariant of $\sigma_{i j}$ (quadratic) and $\alpha_{i j}$ (linear), which ensures the isotropy of the material. 
For a 3D case, if the crack is circular with radius $r$, the compliance tensor is derived by Kachanov (1992):

$$
\mathbf{B}=\frac{32\left(1-v_{0}^{2}\right) r}{3 \pi\left(2-v_{0}\right) E}\left(\mathbf{I}-\frac{v_{0}}{2} \mathbf{n n}\right)
$$

where $\mathbf{I}$ is the second-order identity tensor. The perturbation of potential $\Delta P$ in Equation (8) is:

$$
\Delta P=\frac{16\left(1-v_{0}^{2}\right)}{3\left(2-v_{0}\right) E_{0}}\left\{(\boldsymbol{\sigma} \cdot \boldsymbol{\sigma}): \boldsymbol{\alpha}-\frac{v_{0}}{2} \boldsymbol{\sigma}: \frac{1}{V} \sum_{k}\left(r^{3} \mathbf{n n n n}\right)^{(k)}: \boldsymbol{\sigma}\right\}
$$

Substituting Equation (3) into Equation (15) we have:

$$
\Delta P=\frac{1}{V} \frac{16\left(1-v_{0}^{2}\right)}{3\left(2-v_{0}\right) E_{0}}\left\{\sum_{m}\left[r^{3}\left(\sigma_{i j} \sigma_{j k} n_{j} n_{k}-\frac{v_{0}}{2} \sigma_{i j} \sigma_{k l} n_{i} n_{j} n_{k} n_{l}\right)\right]^{(m)}\right\}
$$

If the crack is of elliptical shape, Kachanov (1992) constructed B by using Eshelby's results for an ellipsoidal inclusion. See also Budiansky and O'Connell (1976).

By using Equations (15) or (16), the effective moduli of cracked rock can now be estimated. For example, for a rock with randomly oriented cracks:

$$
\alpha_{i j}=\frac{\rho}{3} \delta_{i j}
$$

It follows from Equation (16) that:

$$
\Delta P=\frac{16\left(1-v_{0}^{2}\right) \rho}{9\left(2-v_{0}\right) E_{0}}\left\{\left(1-\frac{v_{0}}{5}\right) \sigma_{i j} \sigma_{j i}-\frac{v_{0}}{10} \sigma_{k k}^{2}\right\}
$$

By considering Equations (8), (9) and (18) for uniaxial loading and purely shear loading cases, the corresponding effective moduli can be readily obtained as:

$$
\frac{E}{E_{0}}=\left[1+\frac{16\left(1-v_{0}^{2}\right)\left(10-3 v_{0}\right) \rho}{45\left(2-v_{0}\right)}\right]^{-1}, \frac{G}{G_{0}}=\left[1+\frac{32\left(1-v_{0}\right)\left(5-v_{0}\right) \rho}{45\left(2-v_{0}\right)}\right]^{-1}
$$

For a rock with parallel cracks, say the normal of the crack is in the $x_{1}$ direction:

$$
\boldsymbol{\alpha}=\rho \mathbf{a}_{1} \mathbf{a}_{1}
$$

where $\mathbf{a}_{\mathbf{1}}$ is the unit base vector in the $x_{1}$ direction. Equation (16) now has the following expression:

$$
\Delta P=\frac{16\left(1-v_{0}^{2}\right) \rho}{3\left(2-v_{0}\right) E_{0}}\left\{\sigma_{1 j} \sigma_{j 1}-\frac{v_{0}}{2} \sigma_{11}^{2}\right\}
$$

The corresponding effective moduli in this case are:

$$
\frac{E}{E_{0}}=\left[1+\frac{16\left(1-v_{0}^{2}\right) \rho}{3}\right]^{-1}, \frac{G}{G_{0}}=\left[1+\frac{16\left(1-v_{0}\right) \rho}{3\left(2-v_{0}\right)}\right]^{-1}
$$

\section{Self-consistent scheme by asymptotic approximation of Eshelby's tensor}

In deriving the self-consistent equations which govern the effective elastic constants of composites, two approaches have been used in the literature. The first is based on energy considerations (Budiansky, 1975) 
and the second involves a direct averaging of the components of stress and strain in the constituent phases of the body (Hill, 1965). However, the two methods are actually equivalent to each other.

We consider a solid matrix containing a single ellipsoidal void inclusion with the semi-axes being $a_{1}, a_{2}$ and $a_{3}$, respectively. The matrix is assumed to be placed in an equilibrated state of uniform elastic strain $0^{\circ}$ by external loads. According to Eshelby theory (Eshelby, 1957), the inclusion also reaches a state of strain $\mathbb{E}^{\circ}$ which is uniform inside the void, provided $E^{\infty}$ is uniform. Eshelby termed this strain as the eigenstrain. The relation between the two strains is given by:

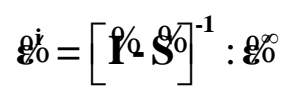

where $\boldsymbol{F}$ is the fourth-order identity tensor and $\boldsymbol{S}^{\prime}$ is the Eshelby's tensor, which depends on the aspect ratios of the ellipsoid and on the Poisson ratio $v$ of the matrix. We assume the semi-axes have the following order of magnitude: $a_{1} \geq a_{2} \geq a_{3} \geq 0$, and define the following two aspect ratios for the ellipsoidal inclusion:

$$
e_{1}=\frac{a_{2}}{a_{1}}, e_{2}=\frac{a_{3}}{a_{2}}
$$

In Voigt's (1928) notation, the exact mathematical definition of the Eshelby tensor for this geometrical configuration can be found as follows Mura (1982):

$$
\boldsymbol{S}=\left[\begin{array}{ll}
\mathbf{X} & \mathbf{0} \\
\mathbf{0} & \mathbf{Z}
\end{array}\right]
$$

where the submatrices $\mathbf{X}$ and $\mathbf{Z}$ have the following expressions:

$$
\begin{gathered}
\mathbf{X}=\frac{1}{8 \pi(1-v)}\left[\begin{array}{ccc}
3 I_{11}+(1-2 v) I_{1} & e_{1}^{2} I_{12}-(1-2 v) I_{1} & e_{1}^{2} e_{2}^{2} I_{13}-(1-2 v) I_{1} \\
I_{12}-(1-2 v) I_{2} & 3 e_{1}^{2} I_{22}+(1-2 v) I_{2} & e_{1}^{2} e_{2}^{2} I_{23}-(1-2 v) I_{2} \\
I_{13}-(1-2 v) I_{3} & e_{1}^{2} I_{23}-(1-2 v) I_{3} & 3 e_{1}^{2} e_{2}^{2} I_{33}+(1-2 v) I_{3}
\end{array}\right] \\
\mathbf{Z}=\frac{1}{8 \pi(1-v)}\left[\begin{array}{ccc}
Z_{11} & 0 & 0 \\
0 & Z_{22} & 0 \\
0 & 0 & Z_{33}
\end{array}\right]
\end{gathered}
$$

and

$$
\begin{gathered}
I_{1}=\frac{4 \pi e_{1}^{2} e_{2}[\mathrm{~F}(p, q)-\mathrm{E}(p, q)]}{\left(1-e_{1}^{2}\right) \sqrt{1-e_{1}^{2} e_{2}^{2}}}, I_{2}=\frac{4 \pi e_{2}\left(1-e_{1}^{2} e_{2}^{2}\right) \mathrm{E}(p, q)}{\left(1-e_{1}^{2}\right)\left(1-e_{2}^{2}\right) \sqrt{1-e_{1}^{2} e_{2}^{2}}}-\frac{4 \pi e_{1}^{2} e_{2} \mathrm{~F}(p, q)}{\left(1-e_{1}^{2}\right) \sqrt{1-e_{1}^{2} e_{2}^{2}}}-\frac{4 \pi e_{2}^{2}}{\left(1-e_{2}^{2}\right)} \\
I_{3}=\frac{4 \pi}{\left(1-e_{2}^{2}\right)}-\frac{4 \pi e_{2} \mathrm{E}(p, q)}{\left(1-e_{2}^{2}\right) \sqrt{1-e_{1}^{2} e_{2}^{2}}}, \quad p=\arcsin \sqrt{1-e_{1}^{2} e_{2}^{2}}, q=\sqrt{\frac{1-e_{1}^{2}}{1-e_{1}^{2} e_{2}^{2}}} \\
Z_{11}=\left(1+e_{1}^{2}\right) I_{12}+(1-2 v)\left(I_{1}+I_{2}\right), Z_{22}=e_{1}^{2}\left(1+e_{2}^{2}\right) I_{23}+(1-2 v)\left(I_{2}+I_{3}\right), \\
\mathrm{F}(p, q)=\int_{0}^{p} \frac{d \alpha}{\sqrt{1-q^{2} \sin ^{2} \alpha}}, \mathrm{E}(p, q)=\int_{0}^{p} \sqrt{1-q^{2} \sin ^{2} \alpha} d \alpha
\end{gathered}
$$




$$
\begin{gathered}
I_{11}=\frac{I_{1}\left(e_{1}^{4} e_{2}^{2}-2 e_{1}^{2} e_{2}^{2}-2 e_{1}^{2}+3\right)+I_{2} e_{1}^{2}\left(e_{1}^{2} e_{2}^{2}-1\right)+I_{3} e_{1}^{2} e_{2}^{2}\left(e_{1}^{2}-1\right)}{3\left(1-e_{1}^{2}\right)\left(1-e_{1}^{2} e_{2}^{2}\right)} \\
I_{22}=\frac{I_{1}\left(1-e_{2}^{2}\right)+I_{2}\left(2 e_{1}^{2} e_{2}^{2}-3 e_{1}^{2}+2-e_{2}^{2}\right)+I_{3} e_{2}^{2}\left(e_{1}^{2}-1\right)}{3 e_{1}^{2}\left(1-e_{1}^{2}\right)\left(1-e_{2}^{2}\right)} \\
I_{33}=\frac{I_{1}\left(1-e_{2}^{2}\right)+I_{2}\left(1-e_{1}^{2} e_{2}^{2}\right)+I_{3}\left(1-2 e_{1}^{2} e_{2}^{2}+3 e_{1}^{2} e_{2}^{4}-2 e_{2}^{2}\right)}{3 e_{1}^{2} e_{2}^{2}\left(1-e_{2}^{2}\right)\left(1-e_{1}^{2} e_{2}^{2}\right)} \\
I_{12}=\frac{I_{2}-I_{1}}{1-e_{1}^{2}}, I_{13}=\frac{I_{3}-I_{1}}{1-e_{1}^{2} e_{2}^{2}}, I_{23}=\frac{I_{3}-I_{2}}{e_{1}^{2}\left(1-e_{2}^{2}\right)}
\end{gathered}
$$

The general Eshelby's tensor in this case is actually a function of $v, e_{1}$ and $e_{2}$ :

$$
\boldsymbol{S}=\boldsymbol{\$}\left(v, e_{1}, e_{2}\right)
$$

When a crack of any type is concerned (such that the aspect ratio $e_{2} \rightarrow 0$ ), the tensor $\mathcal{S}^{\prime}$ can be approximated by its Taylor series with respect to $e_{2}$ about $e_{2}=0$ :

$$
\boldsymbol{S}=\left.\boldsymbol{S}\right|_{e_{2}=0}+\left.\frac{\partial \boldsymbol{S} \mid}{\partial e_{2}}\right|_{e_{2}=0} e_{2}+\mathbf{O}\left(e_{2}^{2}\right)
$$

If we write:

$$
\mathbf{S}=\left.\mathbf{S}\right|_{e_{2}=0} \text { and } \mathbf{M}=\left.\frac{\partial \mathbf{S}}{\partial e_{2}}\right|_{e_{2}=0}
$$

then we have:

$$
\mathbf{S}=\mathbf{S}\left(v, e_{1}\right), \quad \mathbf{M}=\mathbf{M}\left(v, e_{1}\right)
$$

Therefore, if we neglect the higher-order terms other than the first two terms in the right-hand side of Equation (27), the following asymptotic form can be regarded as a good approximation of the Eshelby's tensor for a cracks:

$$
\mathbf{S} \rightarrow \mathbf{S}+\mathbf{M} e_{2}
$$

If penny-shaped cracks are treated such that $a_{1}=a_{2}=r$ and $e_{1}=1$, we further have (Yang et al., 2001):

$$
\mathbf{S}^{p}=\mathbf{S}^{p}(v) \text { and } \mathbf{M}^{p}=\mathbf{M}^{p}(v)
$$

where the superscript $p$ denotes penny-shaped cracks. From the definition of Eshelby's tensor in Equation (25) we know the following limits for $\mathbf{S}^{p}$ when $e_{2} \rightarrow 0$,

$$
\begin{gathered}
S_{2323}^{p}=S_{2332}^{p}=S_{3223}^{p}=S_{3232}^{p}=0.5, \quad S_{1313}^{p}=S_{1331}^{p}=S_{3113}^{p}=S_{3131}^{p}=0.5, \\
S_{3311}^{p}=S_{3322}^{p}=v /(1-v), S_{3333}^{p}=1
\end{gathered}
$$

with all other elements vanishing.

The above expressions are defined in the local coordinate system where the principal axes of the crack coincide with the coordinate directions. Suppose the directional cosines of this local coordinate system with 
respect to a global system are $n_{i}^{(1)}, n_{i}^{(2)}, n_{i}^{(3)}$, respectively. If in the local system the Eshebly's tensor is $\mathbf{S}^{\prime}$, in the global coordinate system the tensor $\mathbf{S}$ can be determined by the following transformation:

$$
S_{i j k l}=n_{i}^{(m)} n_{j}^{(n)} n_{k}^{(p)} n_{l}^{(q)} S_{m n p q}^{\prime}
$$

Note that $n_{i}^{(1)} n_{j}^{(1)}+n_{i}^{(2)} n_{j}^{(2)}+n_{i}^{(3)} n_{j}^{(3)}=\delta_{i j}$.

We now restrict our discussion to the case of penny-shape cracks only. In this case, the global tensor $\mathbf{S}$ can be written in the following form:

$$
S_{i j k l}=\frac{1}{2}\left(\delta_{i k} n_{j} n_{l}+\delta_{i l} n_{j} n_{k}+\delta_{j k} n_{i} n_{l}+\delta_{j l} n_{i} n_{k}-4 n_{i} n_{j} n_{k} n_{l}\right)+\frac{v}{1-v} n_{i} n_{j}\left(\delta_{k l}-n_{k} n_{l}\right)+n_{i} n_{j} n_{k} n_{l}
$$

It can be readily verified that Equation (34) reduces to Equation (32) when $\mathbf{n}=\{0,0,1\}$. $\mathbf{S}$ can be further decomposed into two parts:

$$
\mathbf{S}=\mathbf{S}^{n}+\mathbf{S}^{t}
$$

where:

$$
\mathbf{S}^{n}=\frac{v}{1-v} \mathbf{n n I}+\frac{1-2 v}{1-v} \mathbf{n n n n}, \quad S_{i j k l}^{t}=\frac{1}{2}\left(n_{i} n_{k} \delta_{j l}+n_{i} n_{l} \delta_{j k}+n_{j} n_{k} \delta_{i l}+n_{j} n_{l} \delta_{i k}-4 n_{i} n_{j} n_{k} n_{l}\right)
$$

Analogously, the global tensor $\mathbf{M}$ transformed from a local one has the following expression:

$$
\begin{aligned}
M_{i j k l}= & \frac{\pi(v-2)}{8(1-v)}\left(\delta_{i k} n_{j} n_{l}+\delta_{i l} n_{j} n_{k}+\delta_{j k} n_{i} n_{l}+\delta_{j l} n_{i} n_{k}-4 n_{i} n_{j} n_{k} n_{l}\right) \\
& +\frac{\pi(4 v+1)}{8(1-v)} n_{i} n_{j}\left(\delta_{k l}-n_{k} n_{l}\right)+\frac{\pi(2 v-1)}{8(1-v)} n_{k} n_{l}\left(\delta_{i j}-n_{i} n_{j}\right)+\frac{\pi(2 v-1)}{4(1-v)} n_{i} n_{j} n_{k} n_{l}
\end{aligned}
$$

The energy perturbation produced by a single isolated crack in an infinite medium, as stated by Budiansky and O'Connell (1976), is only influenced by the resolved normal stress $\sigma$ and $\tau$ tangential to the plane of the crack and must be a quadratic function of these stresses. Yang et al. (2001) presented a mathematical derivation of the expression for this energy perturbation based on the asymptotic approximation method. For an isotropic media, an asymptotic expression for the eigenstrain is firstly found in terms of $\mathbf{S}_{n}, \mathbf{S}_{t}$ and the aspect ratio $e_{2}$ :

$$
\varepsilon^{j} \rightarrow \frac{1}{e_{2}}\left(\frac{4(1-v)^{2}}{\pi(1-2 v)} \mathbf{S}_{n}+\frac{4(1-v)}{\pi(2-v)} \mathbf{S}_{t}\right): \varepsilon^{20}
$$

Note that in deriving Equation (38), Equation (37) has been used. The energy perturbation is then obtained by:

$$
\begin{aligned}
\Delta Q=\frac{V}{2} \boldsymbol{\sigma}^{\infty}: \dot{\theta}^{\dot{\delta}} & =\lim _{e_{2} \rightarrow 0} \frac{1}{2} \frac{4 \pi}{3} r^{3} e_{2} \boldsymbol{\sigma}^{\infty}:\left(\frac{1}{e_{2}}\left(\frac{4(1-v)^{2}}{\pi(1-2 v)} \mathbf{S}_{n}+\frac{4(1-v)}{\pi(2-v)} \mathbf{S}_{t}\right): \mathbf{E}^{\infty}\right) \\
& =\frac{1}{2} \frac{4 \pi}{3} r^{3} \boldsymbol{\sigma}^{\infty}:\left(\frac{4(1-v)^{2}}{\pi(1-2 v)} \mathbf{S}_{n}+\frac{4(1-v)}{\pi(2-v)} \mathbf{S}_{t}\right): \mathbf{e}^{\infty} \\
& =\frac{1}{2} \frac{4 \pi}{3} r^{3} \boldsymbol{\sigma}^{\infty}:\left(\frac{4(1-v)^{2}}{\pi(1-2 v)} \mathbf{S}_{n}+\frac{4(1-v)}{\pi(2-v)} \mathbf{S}_{t}\right):\left(\mathbf{C}: \boldsymbol{\sigma}^{\infty}\right)
\end{aligned}
$$


where $\mathbf{C}$ is the compliance tensor of the virgin material, and $\mathbf{C}=-\frac{v}{E} \mathbf{I}^{(2)} \mathbf{I}^{(2)}+\frac{1+v}{E} \mathbf{I}^{(4)}$. It is easy to find that:

$$
\mathbf{S}_{n}: \mathbf{C}=\frac{(1+v)(1-2 v)}{E(1-v)} \mathbf{N}, \mathbf{S}_{t}: \mathbf{C}=\frac{2(1+v)}{E} \mathbf{T}
$$

where the tensors $\mathbf{N}$ and $\mathbf{T}$ are associated with the normal and tangential components of a stress tensor in the direction of $\mathbf{n}$ (Yang et al., 2001):

$$
\mathbf{N}=\mathbf{n n n n}, \mathbf{T}=\frac{1}{2} \mathbf{S}^{t}
$$

Equation (39) can thus be reformulated as:

$$
\Delta Q=\frac{r^{3}}{E}\left[\sigma_{0}^{2} f(v)+\tau_{0}^{2} g(v)\right]
$$

where:

$$
\sigma_{0}^{2}=\boldsymbol{\sigma}^{\infty}: \mathbf{N}: \boldsymbol{\sigma}^{\infty}, \tau_{0}^{2}=\boldsymbol{\sigma}^{\infty}: \mathbf{T}: \boldsymbol{\sigma}^{\infty}, f(v)=\frac{8}{3}\left(1-v^{2}\right), g(v)=\frac{16\left(1-v^{2}\right)}{3(2-v)}
$$

Equation (42) recovers the exact same form as developed by Budiansky and O'Connell (1976). Now we rewrite Equation (42) as:

$$
\begin{aligned}
\Delta Q & =\frac{8 r^{3}\left(1-v^{2}\right)}{3 E}\left[\sigma_{i j} n_{i} n_{j} n_{k} n_{l} \sigma_{k l}+\frac{1}{2(2-v)} \sigma_{i j}\left(n_{i} n_{k} \delta_{j l}+n_{i} n_{l} \delta_{j k}+n_{j} n_{k} \delta_{i l}+n_{j} n_{l} \delta_{i k}-4 n_{i} n_{j} n_{k} n_{l}\right) \sigma_{k l}\right] \\
& =\frac{8 r^{3}\left(1-v^{2}\right)}{3 E}\left[\sigma_{i j} \sigma_{k l} n_{i} n_{j} n_{k} n_{l}+\frac{1}{2(2-v)}\left(4 \sigma_{i j} \sigma_{j k} n_{i} n_{k}-4 \sigma_{i j} \sigma_{k l} n_{i} n_{j} n_{k} n_{l}\right)\right] \\
& =\frac{8 r^{3}\left(1-v^{2}\right)}{3 E}\left[\sigma_{i j} \sigma_{k l} n_{i} n_{j} n_{k} n_{l}+\frac{2}{(2-v)}\left(\sigma_{i j} \sigma_{j k} n_{i} n_{k}-\sigma_{i j} \sigma_{k l} n_{i} n_{j} n_{k} n_{l}\right)\right] \\
& =\frac{16 r^{3}\left(1-v^{2}\right)}{3(2-v) E}\left[\sigma_{i j} \sigma_{j k} n_{i} n_{k}-\frac{v}{2} \sigma_{i j} \sigma_{k l} n_{i} n_{j} n_{k} n_{l}\right]
\end{aligned}
$$

Equation (44) represents the energy perturbation contributed by a single crack. If it is assumed the cracks are non-interacting, such that the energy perturbation for all cracks can be superposed, we are able to sum up Equation (44) for all cracks in the material body and then divide it by the material volume $V$, which leads to the following potential density: $\sum \Delta Q / V$.

It is readily observed that this result is identical with the one in Equation (16) which was derived from a noninteracting method. However, a key point on which the self-consistent method is based is that a crack is placed into a matrix with the effective elastic moduli, not the virgin ones. For randomly distributed cracks, the elastic potential is thus now a function of the effective Young's modulus $\bar{E}$ and effective Poisson's ratio $\bar{v}$, both of which are dependent on the crack density $\rho$. As a result we now have:

$$
\frac{\sum \Delta Q}{V}=\frac{16\left(1-(v(\rho))^{2}\right) \rho}{9(2-v(\rho)) E(\rho)}\left\{\left(1-\frac{v(\rho)}{5}\right) \sigma_{i j} \sigma_{j i}-\frac{v(\rho)}{10} \sigma_{k k}^{2}\right\}
$$

The total elastic potential is then: 


$$
\Delta P=\frac{\sum \Delta Q}{V}=\left(\frac{1+v_{0}}{2 E_{0}}+\frac{16\left(1-v^{2}\right)(5-v) \rho}{45(2-v) E}\right) \sigma_{i j} \sigma_{i j}-\left(\frac{v_{0}}{2 E_{0}}+\frac{8\left(1-v^{2}\right) v \rho}{45(2-v) E}\right) \sigma_{k k}^{2}
$$

Therefore we have:

$$
\left\{\begin{array}{l}
\frac{1}{E_{0}}+\frac{16\left(1-v^{2}\right)(5-v)(10-3 v) \rho}{45(2-v) E}=\frac{1}{E} \\
\frac{1+v_{0}}{E_{0}}+\frac{32\left(1-v^{2}\right)(5-v) \rho}{45(2-v) E}=\frac{(1+v)}{E}
\end{array}\right.
$$

Solving Equation (47) for $E$ and $v$ we can then obtain the effective moduli of the cracked rocks by the selfconsistent scheme, which are essentially softer than the non-interacting results at larger crack densities. It is assumed that the inclusion of cracks in the matrix does not change the Poisson's ratio too much, such that one can safely assume that $v=v_{0}$. In this case, the self-consistent results lead to the following solutions to the elastic moduli:

$$
\frac{E}{E_{0}}=1-\frac{16\left(1-v_{0}^{2}\right)\left(5-v_{0}\right)\left(10-3 v_{0}\right) \rho}{45\left(2-v_{0}\right)}, \frac{G}{G_{0}}=1-\frac{32\left(1-v_{0}\right)\left(5-v_{0}\right) \rho}{45\left(2-v_{0}\right)}
$$

It is interesting to point out that, based on an assumption that the Poisson's ratio does not change significantly due to the presence of the cracks, Berryman et al. (2002) employed a differential scheme and derived the following approximate analytical solutions for a randomly distributed cracked rock:

$$
\frac{E}{E_{0}}=(1-\rho)^{\frac{4\left(1-v_{0}^{2}\right)}{3 \pi e_{2}\left(1-2 v_{0}\right)}} \quad \frac{G}{G_{0}}=(1-\rho)^{\frac{1}{5}\left[1+\frac{8\left(1-v_{0}\right)\left(5-v_{0}\right)}{3 \pi e_{2}\left(2-v_{0}\right)}\right]}
$$

where $e_{2}$ is the aspect ratio defined in Equation (24). Equation (49) takes into account the effect of aspect ratio on the effective moduli.

Figure 1 and Figure 2 present the effective moduli predicted by Equations (19), (48) and (49) for a rock with randomly distributed cracks in the 3D case. Four values of $e_{2}$ are used for Equation (49). As can be seen, Equation (49) predicts an effective Young's modulus whose value is between that given by Equation (19) and Equation (48). The larger is $e_{2}$, the higher is $E$. However, for the effective shear modulus, Equation (49) gives values of $G$ that are close to those obtained from Equations (19) and (48) only when $e_{2}$ is large. When $e_{2}$ is as small as 0.1, Equation (49) gives the smallest value of $G$ of all three predictions.

\section{Discussion and conclusions}

We have used a non-interacting approach based on the crack compliance tensor and a self-consistent approach based on an asymptotic approximation of the Eshelby's tensor, to investigate the problem of the effective elastic properties of cracked rocks. It is shown that the asymptotic method can be used to estimate the effective moduli and can be easily degenerated to the non-interacting approach when the energy perturbation is assumed to be superposable. The non-interacting method is generally known as good approximation when the crack density is low. The same can be said for the self-consistent scheme.

When crack density is high, a well-know problem occurs for the self-consistent scheme (SCS). For porous media with spherical voids the SCS predicts that the effective elastic moduli diminish linearly with void volume fraction until they vanish at $50 \%$, which is in disagreement with experimental results. Similar 
problems are encountered in the SCS results for penny-shaped cracks. The effective Young's modulus diminishes linearly with the crack density parameter and the effective shear modulus nearly so, both moduli abruptly vanishing for a value of crack density parameter at $9 / 16$. Similar behaviour can be observed by the approximation stated in Equation (48) and illustrated in Figures 1 and 2. This is not reasonable since the moduli should vanish asymptotically with increasing crack density. Linear variation with crack density parameter is restricted to small crack density when the cracks do not interact and is not therefore necessarily valid for large crack density.

There are still some issues needing to be resolved in the study of cracked rocks. In a recent comment, Kachanov (2007) pointed out that in deriving the effective elastic properties of cracked solids, the importance of crack shapes and influence of fluid in the cracks and the definition on the crack density parameter in relation with the approximate schemes should be taken into account. Further effort is needed in these areas in the study of cracked rocks.

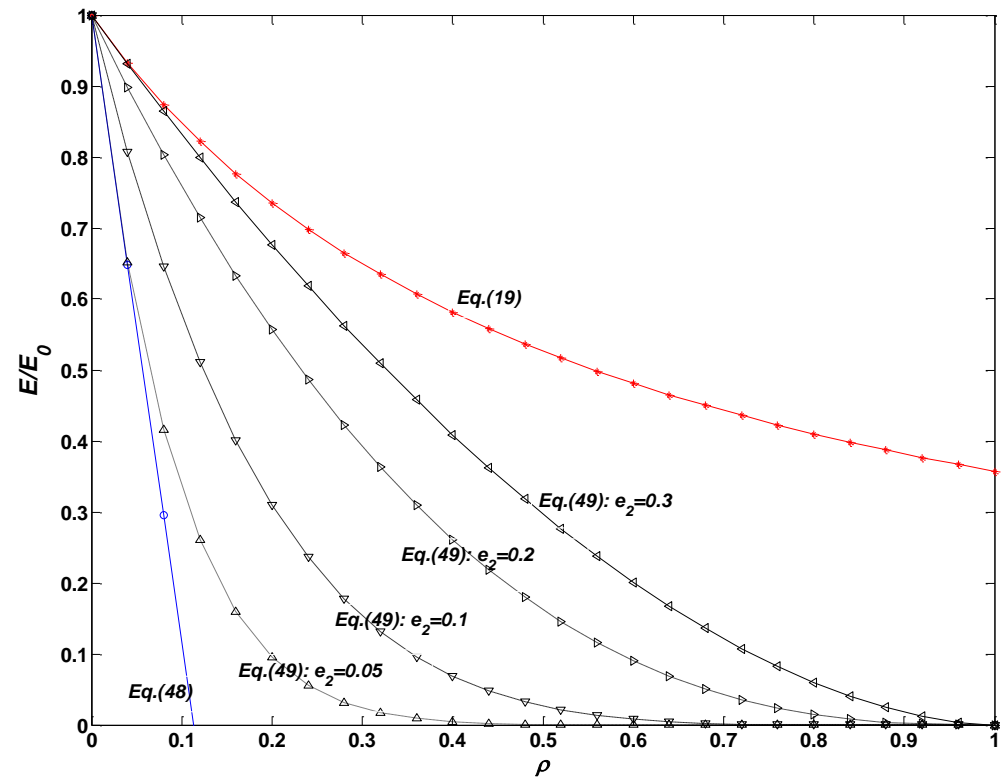

Figure 1 Effective Young's modulus for a rock with randomly distributed cracks in 3D case

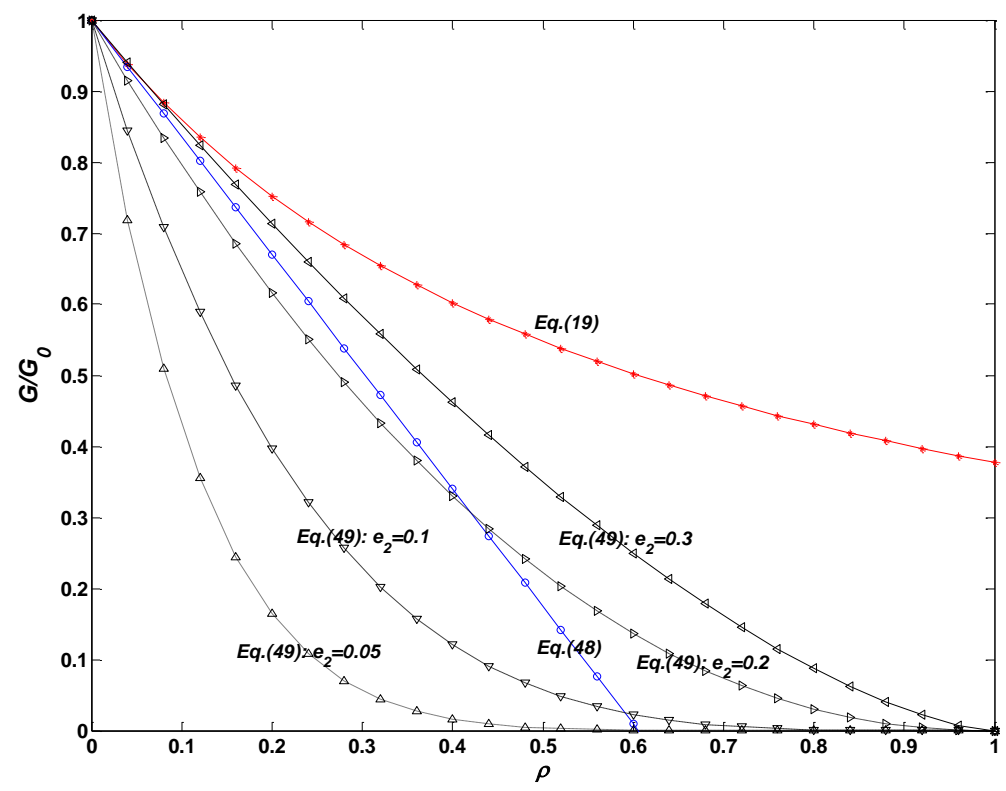

Figure 2 Effective shear modulus for a rock with randomly distributed cracks in 3D case 


\section{References}

Benveniste, Y. (1987) A new approach to the application of Mori-Tanaka's theory in composite materials, Mech. Mater., 6, pp. 147-157.

Berryman, J.G., Pride, S.R. and Wang, H.F. (2002) A differential scheme for elastic properties of rocks with dry or saturated cracks, Geophys., J. Int., 151, pp. 597-611.

Biot, M.A. (1941) General theory of three-dimensional consolidation, J. Appl. Phys., 12, pp. 155-164.

Bristow, J.R. (1960) Microcracks and the static and dynamic elastic constants of annealed and heavily cold-worked metals, British J. Appl. Phys., 11, pp. 81-85.

Budiansky, B. and O’Connell, R.J. (1976) Elastic moduli of a cracked solid, Int. J. Solids Structures, 12, pp. 81-97.

Budiansky, B. (1975) On the elastic moduli of some heterogeneous material. J. Mech. Phys. Solids, 13, pp. $223-227$.

Eshelby, J.D. (1957) The determination of the elastic field of an ellipsoidal inclusion and related problems, Proc. R. Soc. Lond. Ser., A, 241, pp. 376-396.

Hashin, Z. and Shtrikman, S. (1961) Note on a variational approach to the theory of composite elastic materials, J. Franklin Inst., 271, pp. 336-341.

Hashin, Z. and Shtrikman, S. (1962) A variational approach to the theory of elastic behaviour of polycrystals, J. Mech. Phys. Solids, 10, pp. 343-352.

Hashin, Z. and Shtrikman, S. (1963) A variational approach to the theory of the elastic behaviour of multiphase materials, J. Mech. Phys. Solids, 11, pp. 127-140.

Hashin, Z. (1988) The differential scheme and its application to cracked materials, J. Mech. Phys. Solids, 36, pp. 719-734.

Hill, R. (1963) Elastic properties of reinforced solids: some theoretical principles, J. Mech. Phys. Solids, 11, pp. 357-372.

Hill, R. (1965) A self-consistent mechanics of composite materials, J. Mech. Phys. Solids, 13, pp. $213-222$.

Kachanov, M. (1980) Continuum model of medium with cracks, Journal of Engineering Mechanics Division, 106(EM5), pp. 1039-1051.

Kachanov, M. (1987) Elastic solids with many cracks: a simple method of analysis, Int. J. Solids Structures, 23, pp. 23-43.

Kachanov, M. (1992) Effective elastic properties of cracked solids: critical review of some basic concepts, Applied Mechanics Review, 45(10), pp. 304-335.

Kachanov, M. (1994) Elastic solids with many cracks and related problems, Adv. Appl. Mech., 30, pp. $259-445$.

Kachanov, M. (2007) On the effective elastic properties of cracked solids - editor's comments, Int. J. Fract., 146, pp. 295-299.

Mori, T. and Tanaka, K. (1973) Average stress in matrix and average elastic energy of materials with misfitting inclusions, Acta. Met., 21, pp. 571-574.

Mura, T. (1982) Micromechanics of defects in solids, 2nd edn., Martinus Nijhoff, Dordrecht.

Norris, A.N. (1985) A differential scheme for the effective moduli of composites, Mech. Mater., 4, pp. 1-16.

Reuss, A. (1929) Berechnung der Fließgrenze von Mischkristallen auf Grund der Plasizitätsbedingung für Einkristalle, Z. Angrw. Math. Mech., 9, pp. 49-58.

Vavakin, A.S. and Salganik, R.L. (1975) Effective characteristics of nonhomogeneous media with isolated inhomogeneities, Mechanics of Solids, 10, pp. 65-75.

Voigt, W. (1928) Lehrbuch der Kristallphysik, Teubner, Leipzig, p. 962.

Walsh, J.B. (1965a) The effect of cracks on the compressibility of rocks, J .Geophys. Res., 70(2), pp. 381-389.

Walsh, J.B. (1965b) The effect of cracks on unaxial compression of rocks, J. Geophys. Res., 70(2), pp. 399-411.

Yang, Q., Zhou, W.Y. and Swoboda, G. (2001) Asymptotic solutions of penny-shaped inhomogeneities in global Eshelby's tensor, App. Mech. ASME., 68, pp. 740-750.

Zimmerman, R.W. (1991) Elastic moduli of a solid containing spherical inclusions, Mech. Mater., 12, pp. 17-24. 